\title{
Robust Antiwindup Compensation for High-Precision Tracking of a Piezoelectric Nanostage
}

\author{
Pengbo Liu, Peng Yan, Senior Member, IEEE, Zhen Zhang, Member, IEEE, \\ and Hitay Özbay, Senior Member, IEEE
}

\begin{abstract}
Ultrahigh-precision tracking in nanomanipulations poses major challenges for mechanical design as well as servo control, due to the general confliction between the precision requirement and large stroke tracking. The situation is further complicated by input saturation, which is almost inevitable for microactuators. This paper presents a novel control architecture combining a parallel internal-model-based tracking design and a robust antiwindup control structure, such that asymptotic tracking can be achieved for nanoservo systems in the presence of saturation nonlinearity and model uncertainties. For the augmented system with internal-model dynamics, an I/Obased equivalent representation from control (free of saturation) to system output is derived by incorporating the dead-zone nonlinearity, saturation compensation blocks, as well internal-model units. The robustness condition on the saturation compensator is also derived based on the sector bound criterion and an $\mathcal{H}_{\infty}$-optimal design is developed accordingly. The proposed robust antiwindup tracking control architecture is deployed on a customize-designed nanostage driven by a piezoelectric (PZT) actuator, where numerical simulations and real-time experiments demonstrate excellent tracking performance and saturation compensation capability, achieving tracking precision error less than $0.23 \%$.
\end{abstract}

Manuscript received October 9, 2015; revised March 1, 2016; accepted March 24, 2016. Date of publication May 17, 2016; date of current version September 9, 2016. This work was supported in part by the $\mathrm{Na}$ tional Natural Science Foundation of China under Grant 61327003 and Grant 61004004 , in part by the Fundamental Research Funds of Shandong University under Grant 2015JC034, in part by the Open Foundation of the State Key Laboratory of Fluid Power and Mechatronic Systems under Grant GZKF-201413, and in part by the SRF for ROCS, SEM, under Grant 20121028120.

P. Liu is with the Key Laboratory of High-efficiency and Clean Mechanical Manufacture (Shandong University), Ministry of Education, School of Mechanical Engineering, Shandong University, Jinan 250061, China (e-mail: pengbosdu@163.com).

P. Yan is with the Key Laboratory of High-efficiency and Clean Mechanical Manufacture (Shandong University), Ministry of Education, School of Mechanical Engineering, Shandong University, Jinan 250061, China, and also with the School of Automation Science and Electrical Engineering, Beihang University, Beijing 100191, China (e-mail: PengYan2007@gmail.com).

Z. Zhang is with Beijing Key Laboratory of Precision/Ultra-precision Manufacturing Equipments and Control, Tsinghua University, Beijing 100084, China, and also with the State Key Laboratory of Fluid Power and Mechatronic Systems, Zhejiang University, Hangzhou 310027, China (e-mail: zzhang@tsinghua.edu.cn).

$\mathrm{H}$. Özbay is with Department of Electrical and Electronics Engineering, Bilkent University, Ankara 06800, Turkey (e-mail: hitay@bilkent.edu.tr).

Color versions of one or more of the figures in this paper are available online at http://ieeexplore.ieee.org.

Digital Object Identifier 10.1109/TIE.2016.2569060
Index Terms-Antiwindup compensator, internal-model design, nanomanipulator, robustness, trajectory tracking.

\section{INTRODUCTION}

$\mathbf{M}$ ICRO/NANOMANIPULATION has become one of the key enabling technologies for modern precision industries supporting sophisticated servo motion tasks such as nanopositioning or high-performance trajectory tracking, e.g., [1]-[3]. There are abundant results addressing challenging servo control problems associated with nanomanipulations in order to achieve ultrahigh precision motions. Comprehensive literature reviews on control approaches for microactuators and nanosystems are reported in [4] and [5].

Many emerging applications such as track seeking in hard disk drive (HDD) and triangular waveform tracking in atomic force microscope, require nanoprecision trajectory tracking. Theoretical approaches and servo applications of tracking control have been explored with significant research efforts in the past decades, such as [6], [7], and references therein. To address the specific tracking control challenges of nanoservo systems such as the hysteresis nonlinearity of PZT actuators and system uncertainties, more and more results have been reported in recent literatures to improve tracking performance, e.g., robust control [8], adaptive control [9], sliding mode control [10], as well as combinations of feedforward and feedback control [11]. Despite the various control strategies, accurate trajectory tracking for PZT actuated nanostages is still very challenging due to the nonlinear characteristics of PZT actuators and the existence of system uncertainties. Particularly, tracking motions are more likely to trigger saturations for PZT actuators due to their limited strokes. The existence of large disturbances (which is almost inevitable in nanoscale control systems) and initial conditions will deteriorate the situation of saturation, which has significant adverse effects on control system performance and possible mechanical failures [12].

Various control approaches have been developed to deal with actuator saturations, e.g., a parametric discrete-time periodic Lyapunov equation based method in [13], a nested switching control method in [12], an anticipatory antiwindup compensator [14] and a control variable decomposition approach in [15]. Particularly, a decoupled antiwindup structure independent of the saturation-free control scheme was developed by P. F. Weston and I. Postlethwaite in [16]. We call the W-P antiwindup compensator for the seek of brevity. Along this line of research, 
many extensions have been derived such as the robust antiwindup compensator in [17]-[19] and the internal-model-based antiwindup compensator in [20] and [21]. Note that most of existing results on saturation control are discussed for general control systems without considering the specific challenges of tracking control structures and the corresponding performance requirements. It is still an open challenge to develop a systematic control architecture for nanoprecision tracking with the existence of actuator saturations and other model uncertainties.

As one of the most investigated approaches, the internalmodel-based control method has emerged as a fundamental technique for tracking and/or rejecting periodic signals generated by autonomous systems, as it contains a suitable copy of the exosystem to reproduce the desired signals so that the asymptotic tracking is guaranteed [22]. Nevertheless, the internal-model-based tracking theory cannot be effectively applied to nanomanipulating systems due to actuator saturations. It is worth pointing out that although the internal-model-based antiwindup control design has attracted significant research, see [20], [21], and the references therein, it is just a particular case of the W-P antiwindup compensator without tracking capability. In this paper, we propose a novel antiwindup tracking control scheme by combining the parallel internal-model structure [23] with a robust antiwindup compensator. In particular, an I/O-based equivalent representation from control (free of saturation) to system output is derived by incorporating the dead-zone nonlinearity, saturation compensation blocks, as well internal-model units. Furthermore, the robustness condition on the saturation compensator is derived based on the sector bound criterion and an $\mathcal{H}_{\infty}$ optimal design is developed. The result is also a major extension on [16], [24] because the robust design in [16] is based on the unsaturated linear system by assuming (with great simplification) that the overall system with saturation will inherit the same robustness.

The remainder of this paper is organized as follows. In Section II, a systematic control architecture is developed for trajectory tracking by designing a parallel internal-model structure with a robust antiwindup compensator to deal with the saturation nonlinearity and model uncertainties, where guidelines for internal-model units, robust $\mathcal{H}_{\infty}$ stabilizer, and robust antiwindup compensator design are given. A design of a piezodriven nanostage is sketched and its model is established in Section III. As a specific case of a tracking sinusoidal reference, the design of the robust antiwindup tracking controller is described in details in Section IV. The simulations and experimental results for controlling a customize-designed nanostage are demonstrated in Section V, followed by some concluding remarks in Section VI.

\section{Novel INTERnAl-Model Control Structure With ANTIWINDUP COMPENSATION}

The internal-model-based control approach has been explored for servo tracking control in mechatronic systems, e.g., see [23] for reference, where exogenous signals generated by autonomous systems can be immersed into the internal-model unit for asymptotical tracking and/or rejection purposes. Note that high performance tracking of nanomanipulating systems is more

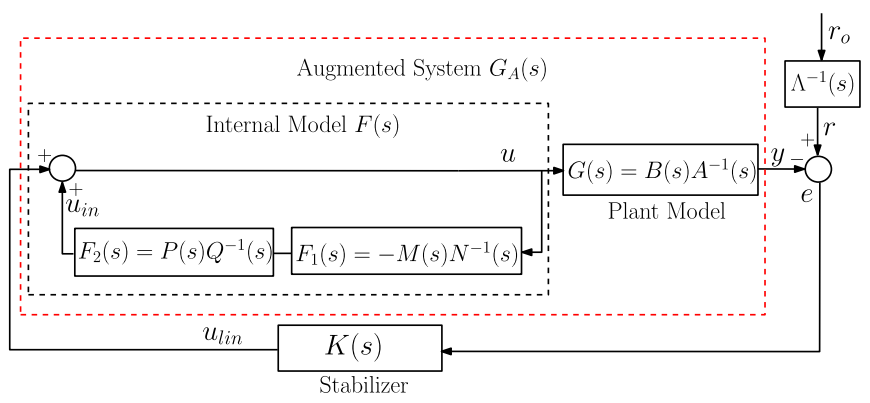

Fig. 1. Block diagram of the parallel internal-model control structure.

challenging due to the existence of hysteresis, system uncertainties, and more importantly, actuator saturations. Actuator saturations deteriorate tracking performance and system stability, thus limit the applicability of existing tracking algorithms. Aiming at high-precision tracking for PZT-driven nanomanipulators, we would like to investigate a robust saturation compensation structure compatible with the internal-model-based tracking design, and explore robustness optimization against various unmodeled dynamics including hysteresis nonlinearity.

\section{A. Parallel Internal-Model Control Structure}

We start with a quick review of a novel parallel internal-model control structure proposed in [23], without considering the actuator saturations. As depicted in Fig. 1, the tracking signals $r(t)$ considered in this paper are assumed to be described by the following exogenous dynamical system:

$$
R(s)=\Lambda(s)^{-1} R_{o}(s)
$$

where $R(s)$ is the Laplace transform of $r(t), \Lambda(s)^{-1}$ represents the dynamics of the exogenous system, and $R_{o}(s)$ is the Laplace transform of $r_{o}(t)$. The disturbance generating signal $r_{o}(t)$ can be typically taken as a short duration pulse or impulse, capturing initial conditions of the exogenous system $\Lambda^{-1}(s)$.

The tracking problem under consideration is to find an error feedback controller such that the following conditions hold.

1) The unforced closed-loop system (i.e., $r(t)=0$ ) is asymptotically stable.

2) The closed-loop system satisfies $\lim _{t \rightarrow \infty} e(t)=0$, for any initial conditions of the plant, and exosystem (1), where $e(t)$ is the tracking error.

We now define $A(s)$ and $B(s), M(s)$ and $N(s), P(s)$ and $Q(s)$ to be the denominator and numerator polynomials of the nominal plant $G(s)$ and internal-model units $F_{1}(s)$ and $F_{2}(s)$, respectively. It is straightforward that the controller design can be decomposed by two ingredients:

1) construction of the internal-model units $F_{1}(s)$ and $F_{2}(s)$;

2) stabilization of the resulting augmented model $G_{A}(s)$.

1) Internal-Model Units: For the parallel internal-model units in Fig. 1, we briefly describe the conditions to achieve asymptotic tracking.

Lemma 1: Suppose that the controller asymptotically stabilizes the unforced closed-loop system, then the controller achieves asymptotic tracking performance if the following 
condition holds:

$$
\left(1+P(s) Q(s)^{-1} M(s) N(s)^{-1}\right)=A(s)^{-1} \Lambda(s) .
$$

Proof. Let

$$
F(s)=F_{1}(s) F_{2}(s)=-P(s) Q(s)^{-1} M(s) N(s)^{-1} .
$$

By simple algebra, we have

$$
E(s)=\frac{(1-F(s)) A(s) \Lambda(s)^{-1} R_{o}(s)}{(1-F(s)) A(s)+B(s) K(s)} .
$$

Hence,

$$
\lim _{t \rightarrow \infty} e(t)=\lim _{s \rightarrow 0} s E(s)=\lim _{s \rightarrow 0} \frac{s(1-F(s)) A(s) \Lambda(s)^{-1} R_{o}(s)}{(1-F(s)) A(s)+B(s) K(s)} .
$$

Assuming that the feedback system is stable, we have a sufficient condition to guarantee asymptotic tracking if $A(s)^{-1}(1-$ $F(s))^{-1}$ includes a copy of the exogenous system. Thus, it is straightforward that $A(s)^{-1}(1-F(s))^{-1}=\Lambda(s)^{-1}$, which is equivalent to (2) and completes the proof.

Remark 1: Note that there are multiple ways to construct $M(s), N(s), P(s)$ and $Q(s)$ satisfying (2). A straightforward one is to set

$$
M(s)=B(s), N(s)=A(s), Q(s)=1
$$

with which the sufficient condition (2) for asymptotic tracking stability can be rewritten as

$$
A(s)+B(s) P(s)=\Lambda(s) .
$$

2) Robust Stabilizer: Once the internal-model units are constructed, we need to design a stabilizer $K(s)$ for the augmented system $G_{A}(s)$ composed of the internal-model units and the control plant. As shown in Fig. 1, we can obtain the augmented system as

$$
G_{A}(s)=\frac{G(s)}{1-F(s)} .
$$

We further assume that the plant model has model uncertainties in additive form described by $\Delta_{G}(s)$. The actual plant model $G_{\Delta}(s)$ can be written as

$$
\begin{array}{r}
G_{\Delta}(s)=G(s)+\Delta_{G}(s)=G(s)(1+\Delta(s)) \\
\left(\left|\Delta_{G}(j \omega)\right|<|W(j \omega)|,|\Delta(j \omega)|<\left|W_{2}(j \omega)\right| \forall \omega \in \Re\right)
\end{array}
$$

where $\Delta(s)=\Delta_{G}(s) / G(s)$ represents the multiplicative uncertainty and $W(s)$ and $W_{2}(s)$ are denoted as the additive uncertainty weighting function and the multiplicative uncertainty weighting function, respectively.

Taking the uncertainties of plant model into account, we have the actual augmented system model as

$$
G_{A \Delta}(s)=\frac{G_{\Delta}(s)}{1-F(s)}=G_{A}(s)(1+\Delta(s)) .
$$

To optimize the stabilizer design with performance requirement and robustness against uncertainties, we would like to formulate the design to a standard mixed sensitivity $\mathcal{H}_{\infty}$ problem [25]. If we denote $S(s)$ and $T(s)$ as the sensitivity and



Fig. 2. W-P antiwindup scheme.

complementary sensitivity of the augmented plant $G_{A}(s)$

$$
\begin{aligned}
S(s) & =\frac{1}{1+G_{A}(s) K(s)} \\
T(s) & =\frac{G_{A}(s) K(s)}{1+G_{A}(s) K(s)} .
\end{aligned}
$$

Our design objective is to find a stabilizing controller $K(s)$ for the following $\mathcal{H}_{\infty}$ optimization problem [26]:

$$
\inf _{K \text { stab. } G_{A}}\left\|\left[\begin{array}{l}
W_{1}(s) S(s) \\
W_{2}(s) T(s)
\end{array}\right]\right\|_{\infty} .
$$

The optimal $\mathcal{H}_{\infty}$ index $\gamma_{\text {opt }}$ and the corresponding $\mathcal{H}_{\infty}$ stabilizer $K_{\text {opt }}(s)$ satisfy

$$
\gamma_{\mathrm{opt}}=\left\|\left[\begin{array}{c}
W_{1}(s)\left(1+G_{A}(s) K_{\mathrm{opt}}(s)\right)^{-1} \\
W_{2}(s)\left(G_{A}(s) K_{\mathrm{opt}}(s)\right)\left(1+G_{A}(s) K_{\mathrm{opt}}(s)\right)^{-1}
\end{array}\right]\right\|_{\infty} .
$$

Remark 2: Note that the performance weighting function $W_{1}(s)$ is usually selected as a low-pass filter in a minimum phase form to improve transient response behaviors and disturbance rejection capability of the closed-loop systems. Meanwhile, the uncertainty weighting function $W_{2}(s)$ can be selected as a minimum phase high-pass filter form to address the plant multiplicative uncertainties.

\section{B. Robust Antiwindup Compensation}

To eliminate the adverse effect of saturation nonlinearities widely observed in microactuators such as piezoelectric actuators, we would like to introduce an antiwindup compensator on top of the proposed internal-model structure. We first recall the W-P compensator depicted in Fig. 2, where the controller output $u$ and the system measurement $y$ will be adjusted based on properly selected $M(s)$ to compensate saturations, as discussed in [16]-[18]. To better accommodate the internal-model structure and handle system uncertainties, we propose a combined antiwindup tracking control architecture, as illustrated in Fig. 3. In case of saturations, the plant input $u_{m}$ will be different from the controller output $u$, and the saturation can be represented by the static and time-invariant relationship between $u$ and $u_{m}$, given by

$$
\operatorname{sat}(u):=u_{m}= \begin{cases}\sigma_{1}, & u \leq \sigma_{1} \\ u, & \sigma_{1}<u<\sigma_{2} \\ \sigma_{2}, & u \geq \sigma_{2}\end{cases}
$$




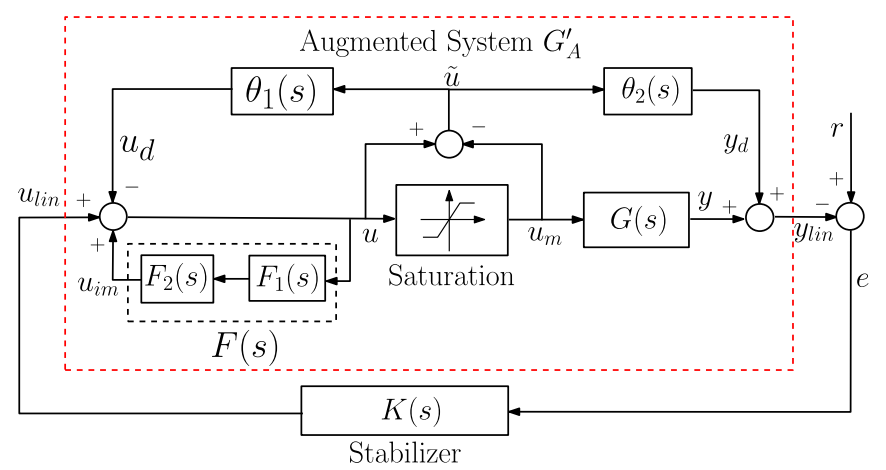

Fig. 3. antiwindup scheme with the internal-model structure.

where $\operatorname{sat}(\cdot)$ is defined as the saturation operator, and the saturation values $\sigma_{1}$ and $\sigma_{2}$ are determined by the nature of actuators.

The antiwindup compensator is driven by the difference between $u$ and $u_{m}$

$$
\tilde{u}=u-u_{m}=u-\operatorname{sat}(u):=d z(u)
$$

where $d z(\cdot)$ is defined as the dead-zone operator.

As depicted in Fig. 3, the antiwindup structure compensates the actuator saturations by adjusting stabilizer output $u_{\text {lin }}$ with $u_{d}$, and adjusting the plant output $y$ with $y_{d}$. If we define $G_{A}^{\prime}=$ $y_{\text {lin }} / u_{\text {lin }}$ as the augmented system of the whole control structure shown in Fig. 3, ideally we would like to have $G_{A}^{\prime}$ identical to $G_{A}$ (see Fig. 1) to fully eliminate the adverse effects on the closed-loop system.

Lemma 2: The augmented system $G_{A}(s)$ shown in Fig. 1 is identical with the augmented system $G_{A}^{\prime}(s)$ shown in Fig. 3, if and only if the following relationship is satisfied:

$$
\frac{\theta_{2}}{1-F+\theta_{1}}=\frac{G}{1-F} .
$$

Proof. From the input-output relationship shown in Fig. 3, we can get

$$
y_{\text {lin }}=\frac{\theta_{2}}{1-F+\theta_{1}} u_{\text {lin }}+\frac{(F-1) \theta_{2}}{1-F+\theta_{1}} u_{m}+G u_{m} .
$$

Sufficiency. If condition (13) holds, we have

$$
\frac{(F-1) \theta_{2}}{1-F+\theta_{1}}=-G \text {. }
$$

Substituting (15) into (14) yields

$$
y_{\text {lin }}=\frac{\theta_{2}}{1-F+\theta_{1}} u_{\text {lin }} .
$$

Therefore, we can calculate the augmented system $G_{A}^{\prime}(s)$ as

$$
G_{A}^{\prime}=\frac{y_{\text {lin }}}{u_{\text {lin }}}=\frac{\theta_{2}}{1-F+\theta_{1}}=\frac{G}{1-F}=G_{A} .
$$

Necessity. Recall the definition of the augmented system $G_{A}(s)$ shown in Fig. 1, we have

$$
y=G_{A} u_{\text {lin }}=\frac{G}{1-F} u_{\text {lin }} .
$$

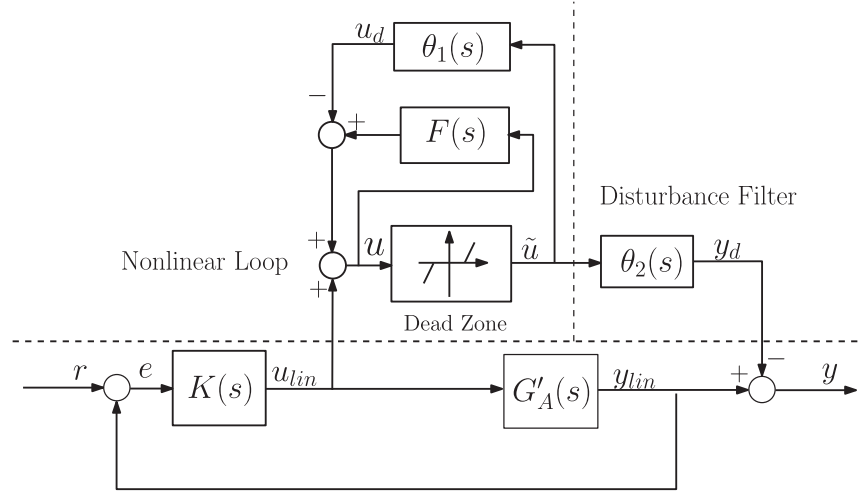

Nominal Linear System

Fig. 4. Equivalent representation of Fig. 3.

Suppose that $G_{A}(s)$ and $G_{A}^{\prime}(s)$ are identical. Observing (14) and (18), we have the relationship as

$$
\frac{\theta_{2}}{1-F+\theta_{1}} u_{\text {lin }}+\frac{(F-1) \theta_{2}}{1-F+\theta_{1}} u_{m}+G u_{m}=\frac{G}{1-F} u_{\text {lin }} .
$$

Obviously, in order to satisfy the aforementioned equation, the following condition have to be satisfied:

$$
\left\{\begin{array}{l}
\frac{\theta_{2}}{1-F+\theta_{1}}=\frac{G}{1-F} \\
\frac{(F-1) \theta_{2}}{1-F+\theta_{1}} u_{m}+G u_{m}=0 .
\end{array}\right.
$$

Therefore, we obtain the necessary condition as

$$
\frac{\theta_{2}}{1-F+\theta_{1}}=\frac{G}{1-F}
$$

and this completes the proof.

Therefore, Fig. 3 can be redrawn as Fig. 4 based on the definition of dead-zone operator (12) and the relationship (13). Similar to the W-P antiwindup compensator, the closed-loop system is divided into three parts: nominal linear system, nonlinear loop, and disturbance filter. Note that the system is governed by the robust tracking control law discussed in previous sections without saturations. In the presence of saturations, the nonlinear loop and disturbance filter of the compensator are activated. Recall the results in [16] and [17]. The stability of the original system with saturation nonlinearity in Fig. 3 is equivalent to the stability of the system with dead-zone operator depicted in Fig. 4. In what follows, we would like to explore the stability condition and performance robustness of the system in Fig. 4 by selecting appropriate $\theta_{1}(s)$ and $\theta_{2}(s)$.

According to the I/O relationships of the nonlinear loop and disturbance filter shown in Fig. 4, we have

$$
\begin{aligned}
\tilde{u} & =d z\left((1-F)^{-1}\left(u_{\text {lin }}-\theta_{1} \tilde{u}\right)\right) \\
y_{d} & =\theta_{2} \tilde{u} .
\end{aligned}
$$

Hence, mapping $\Gamma: u_{\text {lin }} \rightarrow y_{d}$ can be transformed into the structure illustrated in Fig. 5. In order to design $\theta_{1}(s)$ and $\theta_{2}(s)$, 


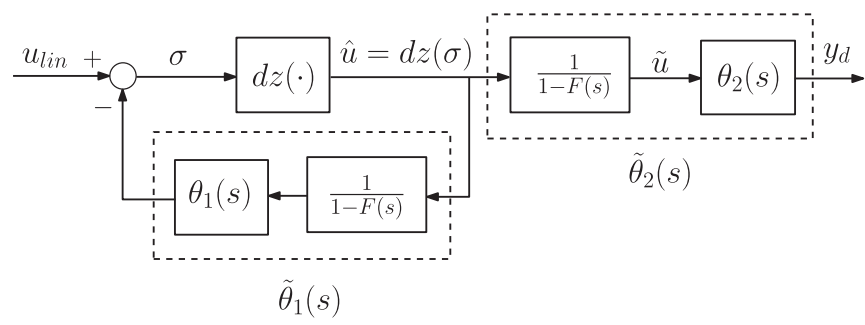

Fig. 5. Equivalent representation of mapping $\Gamma: u_{\text {lin }} \rightarrow y_{d}$.

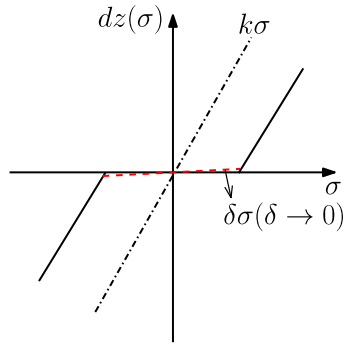

Fig. 6. Illustration of sector bound for dead-zone operator.

we will approximate the dead-zone operator with an operator satisfying the sector bound as illustrated in Fig. 6

$$
\begin{aligned}
& \widehat{d z}(u)= \begin{cases}u-\sigma_{1}, & u \leq \sigma_{1} \\
\delta u(\delta \rightarrow 0), & \sigma_{1}<u<\sigma_{2} \\
u-\sigma_{2}, & u \geq \sigma_{2}\end{cases} \\
& 0<\widehat{d z}(u)<k u, \quad(k=1) .
\end{aligned}
$$

Based on this representation, we can derive a condition to design the antiwindup compensator $\theta_{1}(s)$ and $\theta_{2}(s)$, which guarantees stability of the closed system with dead-zone nonlinearity.

Theorem 1: Consider the robust antiwindup tracking control architecture depicted in Fig. 4, where the dead-zone operator is replaced by $\widehat{d z}(\cdot)$ given in (23). This system is stable if the following conditions are satisfied.

1) The relationship (13) is satisfied;

2) For $\tilde{\theta}_{1}(s):=\theta_{1}(1-F)^{-1}$, there exists an $\alpha>0$ such that

$$
\operatorname{Re}(1+j \alpha \omega) \tilde{\theta}_{1}(j \omega)+1 / k>0
$$

for all $\omega$, where $k$ satisfies $0<u \cdot \widehat{d z}(u)<k u^{2}$.

Proof. According to Lemma 2, if the relationship (13) is satisfied, we have

$$
G_{A}(s)=G_{A}^{\prime}(s) .
$$

Hence, the robust stabilizer $K(s)$ discussed in last subsection can stabilize the nominal linear system shown in Fig. 4.

We further define $\tilde{\theta}_{1}(s):=\theta_{1}(1-F)^{-1}$. Recall Popov criterion [27], [28] that the feedback structure composed of the operator $\widehat{d z}(\cdot)$ and $\tilde{\theta}_{1}(s)$ is stable if condition 2 holds. It is easy to verify that the transfer function $(1-F)^{-1}$ is stable. In addition, the stability of filter $\theta_{2}(s)$ can be guaranteed by (13) with the fact that $\theta_{1}(s)$ and $(1-F)^{-1}$ are stable. Hence, condition 2 [i.e., (25)] is a sufficient condition on the stability of the mapping $\Gamma: u_{\text {lin }} \rightarrow y_{d}$ shown in Fig. 5, which further indicates the stability of the whole system and completes the proof.

Once $\theta_{1}(s)$ is determined by the stability condition of Theorem $1, \theta_{2}(s)$ can be obtained by (13)

$$
\theta_{2}=G\left(1+\frac{\theta_{1}}{1-F}\right)=G\left(1+\tilde{\theta}_{1}\right) .
$$

It is worth noting that the existence of system uncertainties will complicate the aforementioned analysis. As a matter of fact, (13) in Lemma 2 needs to be rewritten as

$$
\frac{\theta_{2 \Delta}}{1-F+\theta_{1}}=\frac{G_{\Delta}}{1-F}
$$

where $G_{\Delta}=G+\Delta_{G}$ presents the system dynamics including additive uncertainty $\Delta_{G}$.

Therefore, $\theta_{2 \Delta}$ can be derived as

$$
\theta_{2 \Delta}=\left(G+\Delta_{G}\right)\left(1+\frac{\theta_{1}}{1-F}\right)=\left(G+\Delta_{G}\right)\left(1+\tilde{\theta}_{1}\right) .
$$

Comparing with $\theta_{2 \Delta}$ derived from (28), $\theta_{2}$ designed for the nominal system without uncertainties has an error of $\Delta_{\theta_{2}}$ defined as

$$
\Delta_{\theta_{2}}=\left|\theta_{2}-\theta_{2 \Delta}\right|=\left|\Delta_{G}\left(1+\tilde{\theta}_{1}\right)\right| .
$$

In order to eliminate the adverse effects caused by plant uncertainties and achieve robust stability and tracking performance, we would like to further minimize $\Delta_{\theta_{2}}$ on admissible sets of $\left(\theta_{1}, \theta_{2}\right)$ satisfying Theorem 1 .

Proposition 1. Consider the robust antiwindup tracking control architecture depicted in Fig. 4, with $\theta_{1}$ and $\theta_{2}$ satisfying Theorem 1, a robust design of $\left(\theta_{1}, \theta_{2}\right)$ with respect to system uncertainty $\Delta_{G}$ can be determined by

$$
\inf \left\|W\left(1+\tilde{\theta}_{1}\right)\right\|_{\infty}
$$

over all $\theta_{1}$ satisfying condition 2 [i.e., (25)], where $W$ is the additive uncertainty bound determined by (6).

\section{Design And Modeling of a Nanostage}

\section{A. Sketch of Nanostage and Experimental Setup}

The design motivation of the piezo-driven nanomanipulator is to support a novel direct writing vacuum evaporation instrument for quantum device fabrications as detailed in [29]. Based on the strict performance specifications of the instrument, the proposed nanostage is expected to satisfy 1) a worksapce of around $100 \mu \mathrm{m}$ and 2) a natural frequency over $300 \mathrm{~Hz}$.

The schematic diagram of the nanomanipulating system is depicted in Fig. 7, where a piezo stack (from Noliac Group) with a free stroke of $25.7 \mu \mathrm{m}$ at $150 \mathrm{~V}$ and a stiffness of $80 \mathrm{~N} / \mu \mathrm{m}$ is used as the actuator. Considering the large workspace requirement, a bridge type displacement amplification mechanism with an amplification ratio of 4.8 is employed to amplify the output displacement of the piezo stack. The central motion platform is connected to the fixed frame through four leaf springs, which constitutes a double four-bar parallelogram guiding mechanism. As a result, the motion of the piezoelectric actuator can be transmitted to the central motion platform accurately. A prototype 


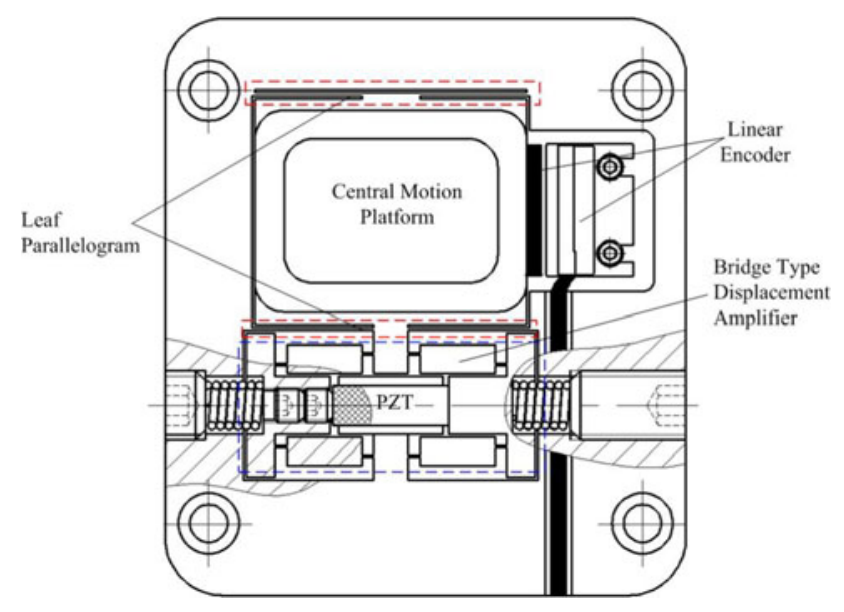

Fig. 7. Schematic diagram of the designed nanostage.

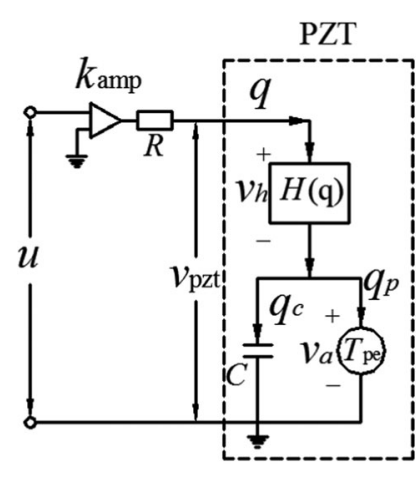

(a)
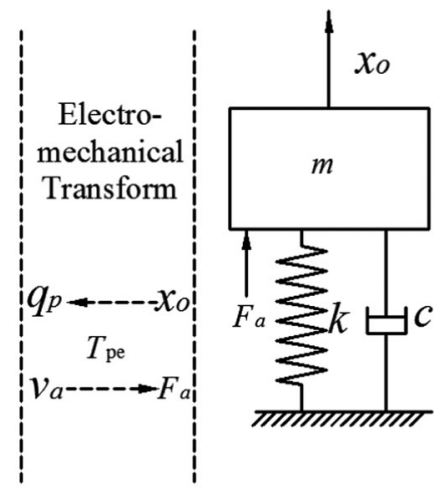

(b)
Fig. 8. Electromechanical model of the piezoelectric-driven nanostage: (a) electrical part and (b) mechanical part.

of the proposed nanostage is monolithically machined by $\mathrm{AL}$ 7075-T6 using the wire electrical discharge machining technique. A linear encoder (from MicroE Systems) with a resolution of $1.2 \mathrm{~nm}$ is instrumented as the displacement sensor to generate real-time position signals. Thus, an experimental apparatus for control and implementation purposes is established, where a high bandwidth voltage amplifier is designed to drive the piezo actuator and feedback control implementations are deployed using a dSPACE R1103 rapid prototyping system. A sampling frequency of $20 \mathrm{kHz}$ is chosen to avoid possible aliasing effects during the experiments and ensure high bandwidth control implementations.

\section{B. Dynamical Modeling and Identification}

Similar to the analysis on the piezoelectric actuators in [30], the dynamic model of the piezoelectric actuator-driven nanostage can be represented by Fig. 8, which consists of the electrical part [see Fig. 8(a)] and the mechanical part [see Fig. 8(b)]. Based on the Kirchhoff law and Newton's law, we can obtain the dynamical model of the piezoelectric-driven nanostage as

$$
\dddot{x}_{o}+a_{2} \ddot{x}_{o}+a_{1} \dot{x}_{o}+a_{0} x_{o}=b_{0} u-b_{1} H(q)
$$
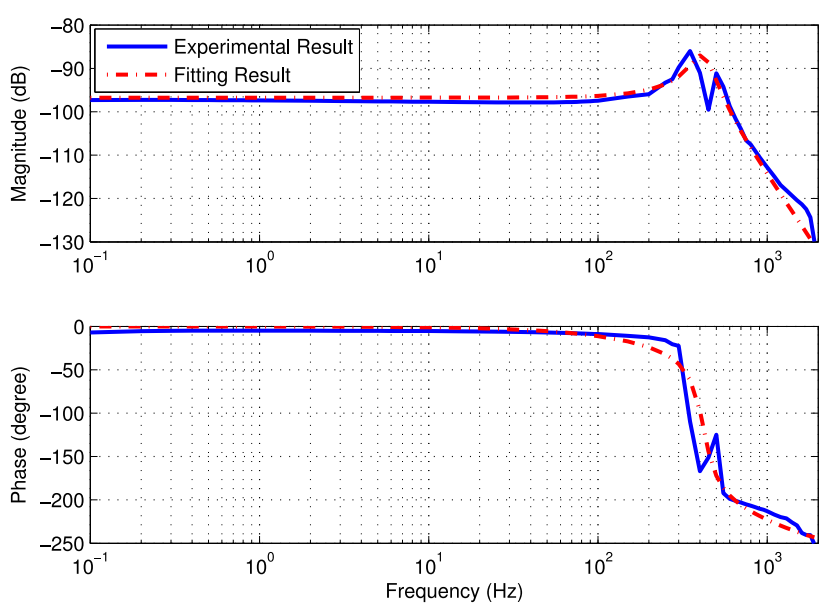

Fig. 9. Open-loop frequency responses.

with

$$
\begin{aligned}
& a_{2}=\frac{c C+m}{m C}, \quad a_{1}=\frac{k C+T_{\mathrm{pe}}^{2}+c}{m C} \\
& a_{0}=\frac{k R C+R T_{\mathrm{pe}}^{2}-T_{p e}^{2}}{m R C^{2}} \\
& b_{0}=\frac{T_{\mathrm{pe}} k_{\mathrm{amp}}}{m R C}, \quad b_{1}=\frac{T_{\mathrm{pe}}}{m R C}
\end{aligned}
$$

where $x_{o}$ is the output displacement of the mechanical part, $m$, $k, c$ are the equivalent mass, stiffness, and damping coefficient of the nanostage, respectively, $k_{\mathrm{amp}}$ and $R$ are the amplification gain and equivalent resistance of the voltage amplifier, $T_{\mathrm{pe}}$ and $C$ are the electromechanical transformer ratio and equivalent capacitance of the piezo actuator, $q$ is the total charge flowing through the circuit, and $H(q)$ represents the nonlinear hysteresis effect.

Apparently the linear portion of the dynamics is a third-order linear system, without considering the nonlinear hysteresis effect $H(q)$. Hence, a real-time DFT algorithm is employed to identify the coefficients $\left(a_{0}, a_{1}, a_{2}\right.$, and $\left.b_{0}\right)$ of the linear model, where swept sine signals are used as excitations and the frequency response data (of the system input and output) are experimentally collected by a real-time data acquisition system. As a result, these coefficients are identified as $\bar{a}_{2}=5810$, $\bar{a}_{1}=1.09 \times 10^{7}, \bar{a}_{0}=3.52 \times 10^{10}$, and $\bar{b}_{0}=5.13 \times 10^{5}$. It clearly demonstrates in Fig. 9 that the third-order model with the estimated parameters can well capture the system's dynamics, by plotting the frequency responses of the experimental data (blue solid line) and identification results (red dash-dot line).

The nanomanipulating system is actually an infinite dimensional system with infinite numbers of flexible modes. In this paper, we consider a third-order system (by using first principle modeling and system identification) and treat all other dynamics as part of the uncertainties for robust design.

\section{Controller Design for the nanostage}

We would like to sketch the design of the proposed control architecture by looking at a trajectory tracking example of 
the nanostage. Assume that the desired reference trajectory is $r(t)=50 \sin (100 \pi t+\pi / 2)+50(\mu \mathrm{m})$. Recall $(1)$, we have the exogenous dynamics as

$$
\Lambda(s)=s^{2}+10000 \pi^{2} .
$$

According to the condition (4) and the identified plant model, the internal-model units can be calculated as

$$
\begin{aligned}
& F_{1}(s)=-\frac{5.13 \times 10^{5}}{s^{3}+5810 s^{2}+1.09 \times 10^{7} s+3.52 \times 10^{10}} \\
& F_{2}(s)=-\left(\frac{\Lambda(s)}{5.13 \times 10^{5}}-\frac{1}{F_{1}(s)}\right) .
\end{aligned}
$$

The robust stabilizer can be synthesized for the augmented system $G_{A}(s)$ based on the requirements on robustness and performance. Following the guidelines of Remark 2 on selecting the weighting function $W_{1}(s)$ and $W_{2}(s)$, we can determine

$$
\begin{aligned}
& W_{1}(s)=\frac{85}{(s+0.01)^{2}} \\
& W_{2}(s)=\frac{4.49 \times 10^{-5}\left(s^{2}+251.67 s+98908.69\right)}{s^{2}+722.57 s+5.22 \times 10^{7}} .
\end{aligned}
$$

Note that the weighting function $W_{1}(s)$ and $W_{2}(s)$ determine the shapes of sensitivity function $S(s)$ and complementary sensitivity function $T(s)$. Topically, $W_{1}(s)$ is chosen to have an integral action to achieve good disturbance rejection performance at low frequencies. Meanwhile, $W_{2}(s)$ is chosen to have high pass to accommodate system uncertainties at high frequencies. By utilizing the MATLAB function "mixsyn", we can apply (10) and derive the following six-order robust stabilizer based on the mixed sensitivity optimization approach:

$$
\begin{aligned}
& 2.47 \times 10^{7} s^{5}+5.04 \times 10^{10} s^{4}+1.31 \times 10^{15} s^{3} \\
& K(s)=\frac{+1.70 \times 10^{18} s^{2}+1.29 \times 10^{20} s+1.68 \times 10^{23}}{s^{6}+1.19 \times 10^{5} s^{5}+7.15 \times 10^{9} s^{4}+5.07 \times 10^{13} s^{3}} \\
& +2.45 \times 10^{17} s^{2}+4.90 \times 10^{15} s+2.45 \times 10^{13}
\end{aligned}
$$

and the optimal $\mathcal{H}_{\infty}$ index $\gamma_{\text {opt }}=3.28 \times 10^{-5}$.

Now that we are ready to consider the robust antiwindup compensator design. Recall condition (2) in Theorem 1, we choose $\tilde{\theta}_{1}$ as

$$
\tilde{\theta}_{1}=\frac{\gamma}{(1+\alpha s)(1+\beta s)}, \quad \alpha>0, \beta>0 .
$$

Hence, (25) can be rewritten as

$$
\frac{\gamma}{1+\beta^{2} \omega^{2}}>-\frac{1}{k}, \quad k=1 .
$$

It is obvious that if $\gamma>-\frac{1}{k}$, the aforementioned inequation is satisfied.

According to Proposition 1, we define a function $f(\cdot)$ as

$$
f(\gamma)=\left\|W(s)\left(1+\frac{\gamma}{(1+\alpha s)(1+\beta s)}\right)\right\|_{\infty} .
$$

We need to determine the optimal values of $\gamma, \alpha$, and $\beta$ to minimize the function $f(\gamma)$. The uncertainty weighting func-

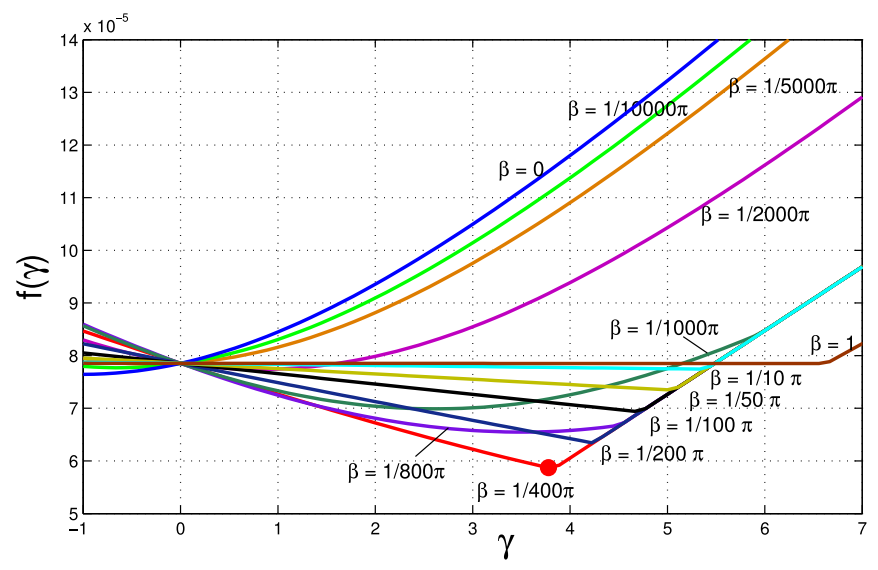

Fig. 10. Curves of function $f$ with different $\beta$.

tion $W(s)$ (to bound the additive uncertainty $\Delta(s)$ ) can be experimentally determined by observing the discrepancy between the experimental measured dynamics and the nominal model.

In this particular design, $W(s)$ is selected in the following form:

$$
W(s)=\frac{k_{0}(1+\alpha s)}{s^{3}+\alpha_{2} s^{2}+\alpha_{1} s+\alpha_{0}}
$$

such that the optimization problem of (39) can be simplified by canceling the term $1+\alpha s$. The parameters of (40) are experimentally determined as $\alpha=1 / 200 \pi, k_{0}=75920$, $\alpha_{0}=6.27 \times 10^{9}, \alpha_{1}=6.83 \times 10^{6}$, and $\alpha_{2}=298.41$.

Moreover, the curves of the function $f$ values versus $\gamma$ with different $\beta$ are plotted in Fig. 10. It can be determined that the function $f$ takes the minimum value when $\gamma=3.78$ and $\beta=1 / 400 \pi$. With this, the parameters $\alpha, \beta$, and $\gamma$ in (39) are determined to achieve the minimal value of function $f(\gamma)$.

We further recall Fig. 5, (26) and (37), and derive the antiwindup compensator $\theta_{1}(s)$ and $\theta_{2}(s)$ as

$$
\begin{array}{r}
\theta_{1}(s)=\frac{2.98 \times 10^{6}\left(s^{2}+98696.04\right)}{s^{5}+7692.01 s^{4}+2.26 \times 10^{7} s^{3}+6.03 \times 10^{10} s^{2}} \\
+7.50 \times 10^{13} s+2.78 \times 10^{16} \\
\theta_{2}(s)=\frac{5.12 \times 10^{5} s^{2}+9.66 \times 10^{8} s+1.93 \times 10^{12}}{s^{5}+7692.01 s^{4}+2.26 \times 10^{7} s^{3}+6.03 \times 10^{10} s^{2}} \\
+7.50 \times 10^{13} s+2.78 \times 10^{16}
\end{array}
$$

Now that we determined the internal-model components $F_{1}(s), F_{2}(s)$, the robust antiwindup components $\theta_{1}(s), \theta_{2}(s)$, and the robust stabilizer $K(s)$, respectively. With this, the overall controller can be derived by employing the antiwindup tracking control structure in Fig. 3.

\section{SimulationS AND IMPLEMENTATIONS}

The experimental apparatus is established as depicted in Fig. 11, where the nanomanipulating system is mounted to a floatation platform for the vibration suppression purpose. Note that the displacement measurement for high-precision tracking is challenging due to the stroke limitation on sensors with 

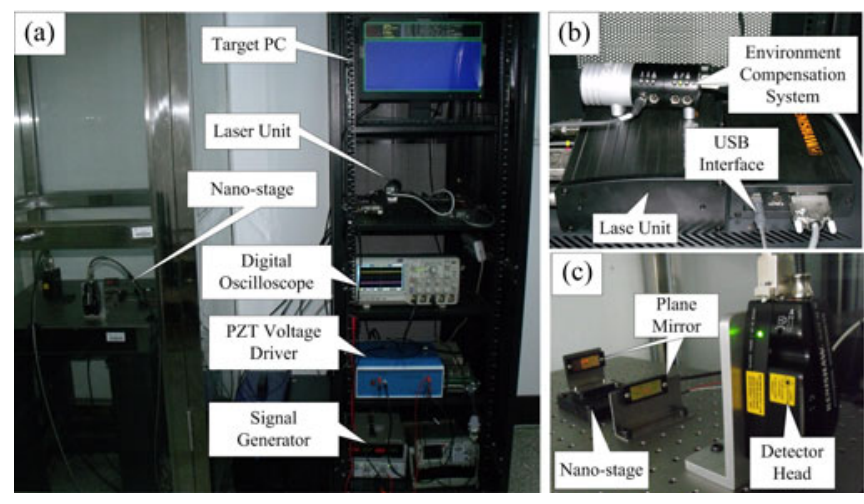

Fig. 11. (a) Experimental setup. (b) Details of laser interferometer. (c) Prototype of the piezoelectric manipulating stage.

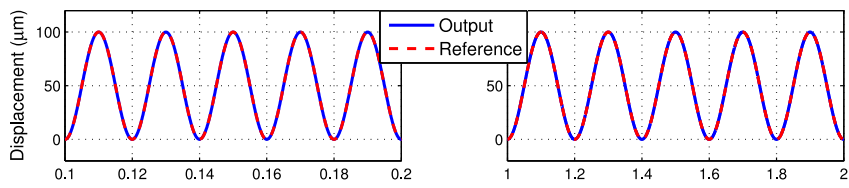

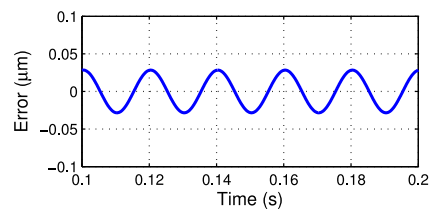

(a)



(b)
Fig. 12. Simulated tracking performance without saturations. (a) $50 \mathrm{~Hz}$ and (b) $5 \mathrm{~Hz}$.

nanoscale resolution. Therefore, a RENISHAW laser interferometer is installed for real-time displacement measurement. The details of the nanomanipulating system can be referred to the description in Section III-A.

Based on the proposed control architecture, some numerical simulations and hardware-in-loop implementations are comprehensively conducted to evaluate the tracking performance in various scenarios. Tracking examples with low-frequency $(5 \mathrm{~Hz}$ in this particular case) and high-frequency (50 Hz in this particular case) references are both tested, where the cases with and without robust antiwindup control scheme are studied and compared.

It is also interesting to compare the proposed robust antiwindup compensation mechanism to other well-known saturation compensation schemes. In particular, we design the W-P antiwindup compensator [16] on top of the internal-model tracking controller illustrated in Fig. 1, for the same tracking problem. Moreover, an adaptive internal-model-based antiwindup structure proposed in [20] is also designed for comparison purpose.

\section{A. Simulation Results}

We start with the case of tracking a sinusoidal reference without saturations. As clearly demonstrated in Fig. 12, the proposed algorithm achieves good performance with less than $0.057 \%$ tracking precision at the frequency of $50 \mathrm{~Hz}$ and less than $0.03 \%$ tracking precision at the frequency of $5 \mathrm{~Hz}$. Since
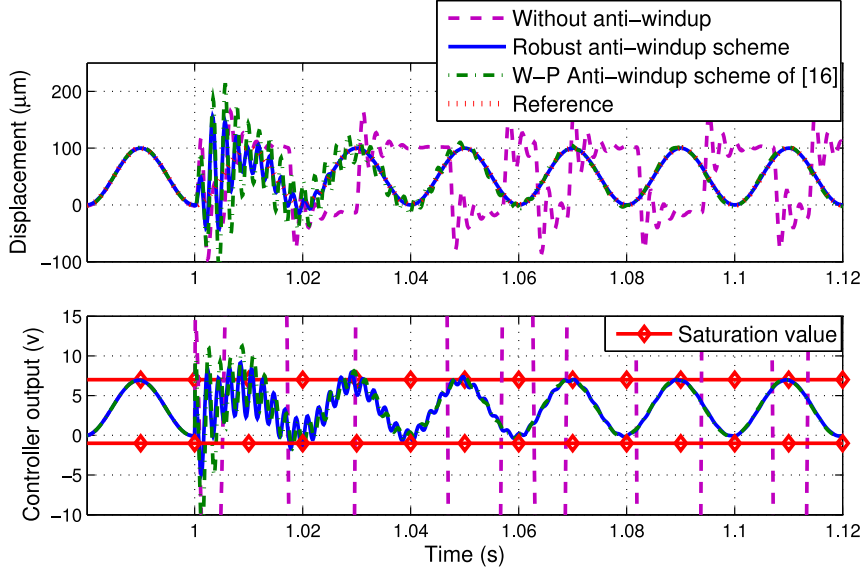

Fig. 13. Simulated tracking performance with input saturation.
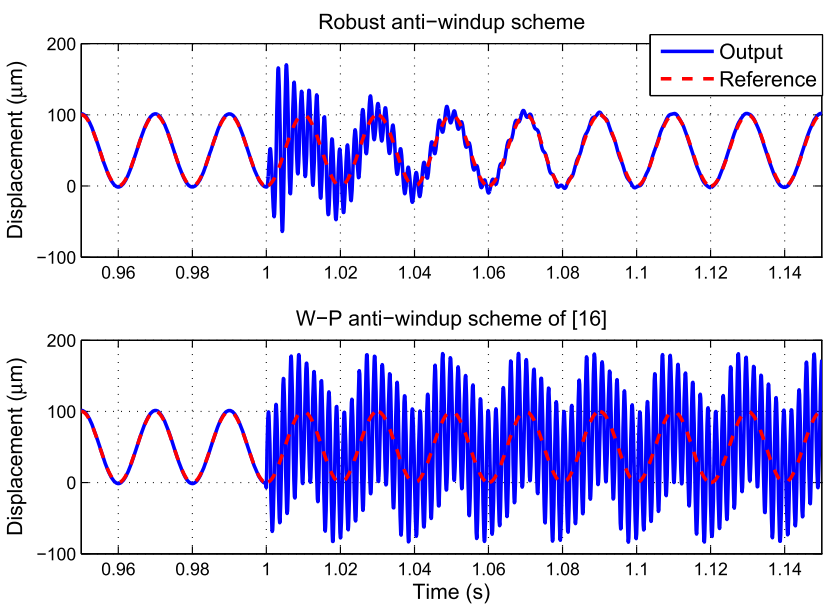

Fig. 14. Simulation results in the case of system parameters variation.

it is more challenging to track high-frequency signals, we will focus on the simulations and experiments for $50 \mathrm{~Hz}$ tracking examples in what follows.

Furthermore, a periodic interference is introduced on top of the control signal to occasionally trigger PZT actuator saturations. As shown in Fig. 13, the simulations demonstrate that the internal-model-based tracking controller without antiwindup compensator cannot track the desired trajectory against saturations, where huge oscillations of control signal and system output are observed. However, with the action of the robust antiwindup compensator, the commanded input converges and the system output achieves asymptotic tracking. It is also clear from Fig. 13 that the proposed robust antiwindup compensator shows smaller oscillations of system output than the W-P antiwindup compensator.

In order to further verify the robustness of the proposed control architecture, we change the model parameters and generate simulation results as depicted in Fig. 14. It is straightforward that the proposed robust antiwindup tracking controller can still achieve asymptotic tracking in spite of longer oscillating time, while the W-P method cannot track the reference trajectory. 


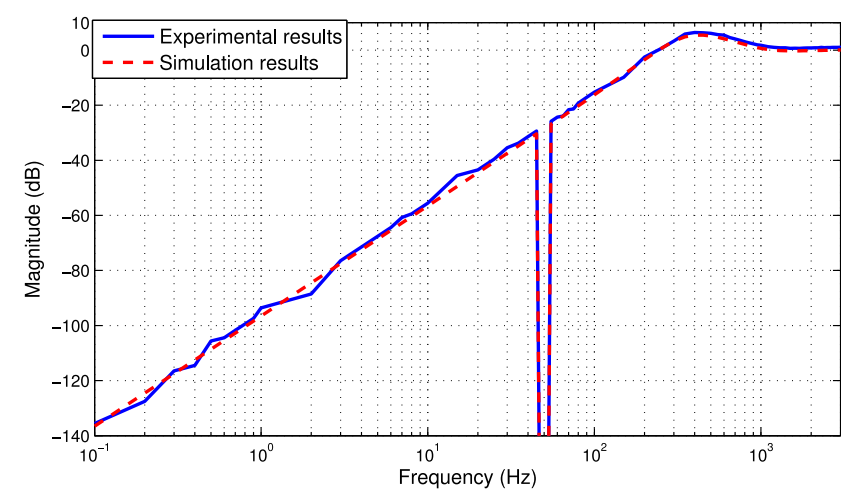

Fig. 15. Frequency response of the sensitivity function.

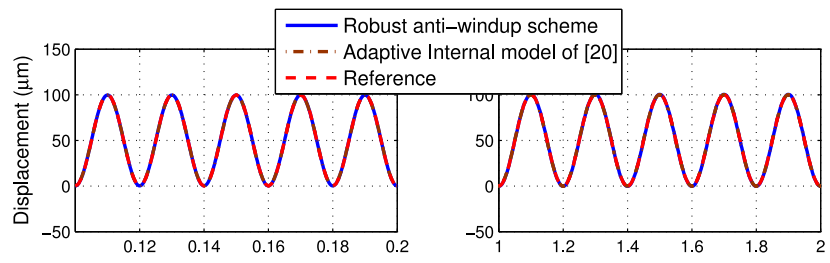

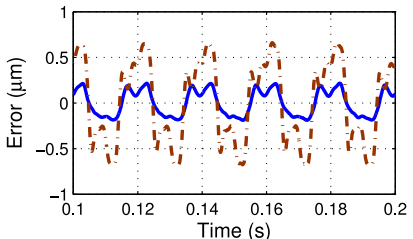

(a)

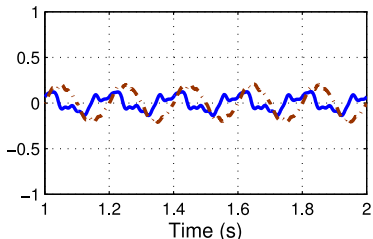

(b)
Fig. 16. Experimental results of tracking without saturations. (a) $50 \mathrm{~Hz}$ and (b) $5 \mathrm{~Hz}$.

TABLE I

Tracking Performance Without InPUt Saturations $(r(t)=50 \sin (2 \pi f t+\pi / 2)+50(\mu \mathrm{m}))$

\begin{tabular}{llcc}
\hline \hline$f(\mathrm{~Hz})$ & \multicolumn{1}{c}{ Controller } & $e_{m}(\mathrm{~nm})$ & $\mathcal{L}_{2}[e](\mathrm{nm})$ \\
\hline 50 & Robust antiwindup scheme & 231.8 & 141.1 \\
& Adaptive internal model of [20] & 660.6 & 447.3 \\
5 & Robust antiwindup scheme & 163.9 & 82.4 \\
& Adaptive internal model of [20] & 211.9 & 140.8 \\
\hline \hline
\end{tabular}

The comparison results demonstrate that the proposed control architecture is more robust than the $\mathrm{W}-\mathrm{P}$ compensator.

\section{B. Experimental Results}

The proposed control architecture is also developed and implemented in real time on the piezoelectric-driven nanostage described in Fig. 11 with the sampling frequency of $20 \mathrm{kHz}$. Before presenting the tracking results, we collected the closedloop frequency response (sensitivity) to examine the closed-loop system dynamics. As depicted in Fig. 15, the experimental frequency responses (blue line) and simulation results (red dash) agree well with each other, where closed-loop bandwidth of around $250 \mathrm{~Hz}$ and a signature of perfect tracking at $50 \mathrm{~Hz}$ are demonstrated. Note that oscillations of the experimental results at low frequencies are due to the small amplitudes of the output
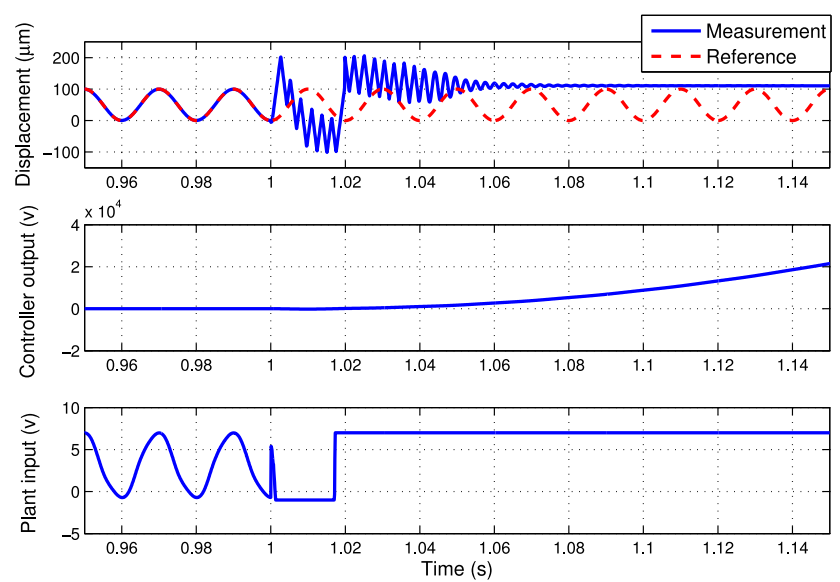

Fig. 17. Experimental results of the internal-model-based controller with input saturation.
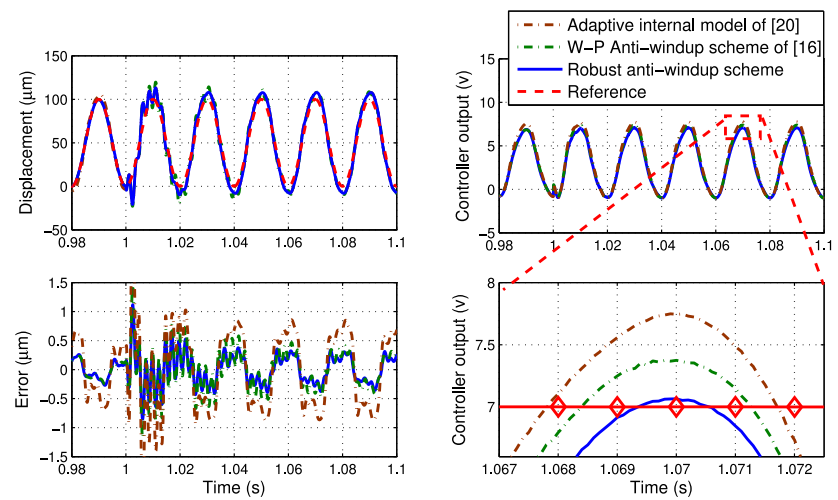

Fig. 18. Experimental results of the saturation compensation.

TABLE II

Tracking PERFormance With InPUt SATURATIONS

$(r(t)=50 \sin (100 \pi t+\pi / 2)+50(\mu \mathrm{m}))$

\begin{tabular}{lccc}
\hline \hline $\begin{array}{l}\text { Performance } \\
\text { indices }\end{array}$ & $\begin{array}{c}\text { Robust } \\
\text { antiwindup }\end{array}$ & $\begin{array}{c}\text { W-P antiwindup } \\
\text { scheme of [16] }\end{array}$ & $\begin{array}{c}\text { Adaptive internal } \\
\text { model of [20] }\end{array}$ \\
\hline$e_{m}(\mu \mathrm{m})$ & 1.12 & 1.46 & 3.17 \\
$\mathcal{L}_{2}[e](\mathrm{nm})$ & 235.1 & 303.6 & 650.4 \\
$\operatorname{sat}[u]_{m}(\mathrm{~V})$ & 0.07 & 0.39 & 0.75 \\
\hline
\end{tabular}

signal. In order to measure the quality of the control algorithm, we define the following indices.

1) Finite-time maximal error in the sense of $\mathcal{L}_{\infty}: e_{m}=$ $\max _{t_{0} \leq t \leq t_{0}+T_{f}}\{|e|\}$, which is the maximal absolute value of the tracking error $e(t)$ during the time interval of $T_{f}$ starting from $t_{0}$.

2) Finite-time average error in the sense of $\mathcal{L}_{2}: \mathcal{L}_{2}[e]=$ $\sqrt{\left(1 / T_{f}\right) \int_{t_{0}}^{t_{0}+T_{f}}|e|^{2} d t}$, which demonstrates the average tracking performance.

3) Finite-time maximal saturation level in the sense of $\mathcal{L}_{\infty}: \operatorname{sat}[u]_{m}=\max _{t_{0} \leq t \leq t_{0}+T_{f}}\left(u(t)-\sigma_{1}\right)$, which is the maximal value of the control effort $u(t)$ exceeding the saturation value $\sigma_{1}$. 


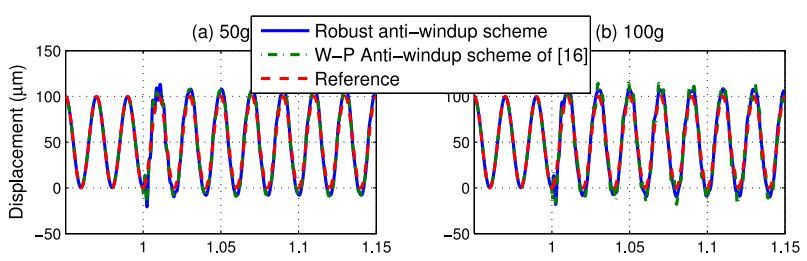

(c) $150 \mathrm{~g}$
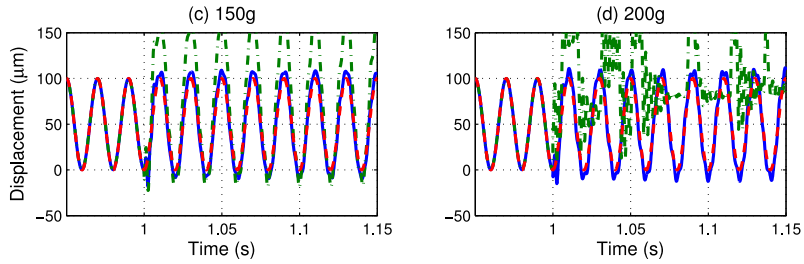

(a)



(c) $150 \mathrm{~g}$
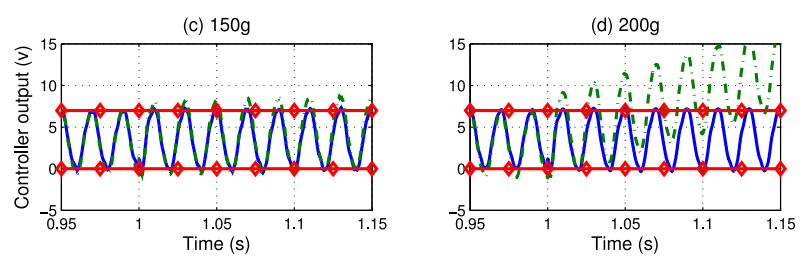

(b)

Fig. 19. Experimental results of the tracking performance in the case of load change. (a) System output. (b) Controller output.

As depicted in Fig. 16, similar good tracking performance is achieved in experiments with the same reference trajectories as simulations. The calculated tracking performance indices are listed in Table I, where excellent tracking performance is achieved without saturations. In particular, the proposed controller can achieve average tracking precision of $0.23 \%$ at the frequency of $50 \mathrm{~Hz}$ and $0.13 \%$ at $5 \mathrm{~Hz}$, while the adaptive internal-model structure is $0.73 \%$ at $50 \mathrm{~Hz}$ and $0.22 \%$ at $5 \mathrm{~Hz}$.

We further add the same periodic interference on top of the control signal to test the saturation compensation performance, similar to the simulations. As depicted in Fig. 17, the internalmodel-based tracking controller without antiwindup compensator cannot stabilize the system when input saturation occurs. Comprehensive comparisons of the proposed control structure with other methods (see[16] and [20]) are illustrated in Fig. 18, where all the schemes can handle saturations well. However, the data of Table II show that the proposed robust antiwindup compensator has the best performance with the average tracking precision of $0.38 \%$, while the W-P method and the adaptive internal-model scheme are $0.49 \%$ and $1.06 \%$, respectively. In the meanwhile, the zoom-in plot in Fig. 18 clearly illustrates that the control behavior of proposed method better matches the saturation bound than that of [16] and [20]. The antiwindup performance index of the robust antiwindup compensator is smallest with sat $[u]_{m}=0.07 \mathrm{~V}$, while the W-P compensator and the adaptive internal-model scheme are 0.39 and $0.75 \mathrm{~V}$, respectively.
To further verify the robustness of the proposed control architecture, different loads are imposed on the nanostage, and comparison experiments are conducted between the proposed robust antiwindup compensator and the W-P approach. As depicted in Fig. 19, the comparison results clearly demonstrate that the proposed antiwindup compensator is more robust than the W-P compensator against system uncertainties. As a matter of fact, when external loads (uncertainties) (ranging from 0 to $200 \mathrm{~g}$ ) are applied, the controller outputs are well compensated by the proposed control approach within the saturation bound, and acceptable tracking performance is achieved. However, the controller output of the W-P antiwindup compensator seriously triggers the saturation, resulting in significant oscillations once the load exceeds $150 \mathrm{~g}$.

\section{CONCLUSION}

For the purpose of high-precision trajectory tracking of nanomanipulating systems with actuator saturations and unmodeled uncertainties, we proposed a novel control architecture combining a parallel internal-model control structure with a robust antiwindup compensator. Stability and robustness conditions of the resulting closed-loop systems were analyzed and controller design guidelines were provided. The control algorithm was also applied to the designed piezo-driven nanostage where the overall control design procedure was detailed with trajectory tracking examples. Simulations and real-time experiments demonstrated excellent performance with tracking precision error less than $0.23 \%$, and outperformed existing methods by better robustness and capability of saturation compensation.

\section{REFERENCES}

[1] J. M. Wu, K. C. Huang, M. L. Chiang, and M. Y. Chen, "Modeling and controller design of a precision hybrid scanner for application in large measurement-range atomic force microscopy," IEEE Trans. Ind. Electron., vol. 61, no. 7, pp. 3704-3712, Jul. 2014.

[2] P. Liu, P. Yan, and Z. Zhang, "Design and analysis of an X-Y parallel nanopositioner supporting large-stroke servomechanism," J. Mech. Eng. Sci., vol. 229, no. 2, pp. 364-376, Feb. 2015.

[3] H. Tang and Y. Li, "Development and active disturbance rejection control of a compliant micro-/nanopositioning piezostage with dual mode," IEEE Trans. Ind. Electron., vol. 61, no. 3, pp. 1475-1492, Mar. 2014.

[4] S. Devasia, E. Eleftheriou, and S. O. R. Moheimani, "A survey of control issues in nanopositioning," IEEE Trans. Control Syst. Technol., vol. 15, no. 5, pp. 802-823, Sep. 2007.

[5] G. M. Clayton, S. Tien, K. K. Leang, Q. Zou, and S. Devasia, "A review of feedforward control approaches in nanopositioning for highspeed SPM," ASME J. Dyn. Syst., Meas., Control, vol. 131, no. 6, pp. 061101-1-061101-19, Nov. 2009.

[6] J. She, L. Zhou, M. Wu, J. Zhang, and Y. He, "Design of a modified repetitive-control system based on a continuous-discrete 2D model," $\mathrm{Au}$ tomatica, vol. 48, no. 5, pp. 844-850, May 2012.

[7] K. Kim and Q. Zou, "A modeling-free inversion-based iterative feedforward control for precision output tracking of linear time-invariant systems," IEEE/ASME Trans. Mechatronics, vol. 18, no. 6, pp. 1767-1777, Dec. 2013.

[8] A. Sebastian and S. M. Salapaka, "Design methodologies for robust nano-positioning," IEEE Trans. Control Syst. Technol., vol. 13, no. 6 , pp. 868-876, Nov. 2005.

[9] H. Liaw and B. Shirinzadeh, "Robust adaptive constrained motion tracking control of piezo-actuated flexure-based mechanisms for micro/nano manipulation," IEEE Trans. Ind. Electron., vol. 58, no. 4, pp. 1406-1415, Apr. 2011. 
[10] Q. Xu, "Digital sliding-mode control of piezoelectric micropositioning system based on input-output model," IEEE Trans. Ind. Electron., vol. 61, no. 10, pp. 5517-5526, Oct. 2014.

[11] G. Song, J. Zhao, X. Zhou, and J. D. Abreu-Garcia, "Tracking control of a piezoceramic actuator with hysteresis compensation using inverse Preisach model," IEEE/ASME Trans. Mechatronics, vol. 10, no. 2 , pp. 198-209, Apr. 2005

[12] J. Zheng and M. Fu, "Saturation control of a piezoelectric actuator for fas settling-time performance," IEEE Trans. Control Syst. Technol., vol. 21, no. 1, pp. 220-228, Jan. 2013

[13] B. Zhou, G. Duan, and Z. Lin, "A parametric periodic Lyapunovequation with application in semi-global stabilization of discrete-time periodic systems subject to actuator saturation," Automatica, vol. 47, no. 2, pp. 316-325, Feb. 2011

[14] X. Wu and Z. Lin, "Dynamic anti-windup design in anticipation of actuator saturation," Int. J. Robust Nonlinear Control, vol. 24, no. 2, pp. 295-312, Jan. 2014.

[15] A. Rauh, L. Senkel, and H. Aschemann, "Interval-based sliding mode control design for solid oxide fuel cells with state and actuator constraints," IEEE Trans. Ind. Electron., vol. 68, no. 8, pp. 5208-5217, Aug. 2015.

[16] P. F. Weston and I. Postlethwaite, "Linear conditioning for systems containing saturating actuators," Automatica, vol. 36, no. 9, pp. 1347-1354, Sep. 2000.

[17] M. C. Turner, G. Herrmann, and I. Postlethwaite, "Incorporating robustness requirements into antiwindup design," IEEE Trans. Autom. Control, vol. 52, no. 10, pp. 1842-1855, Oct. 2007.

[18] G. Li, G. Herrmann, D. P. Stoten, J. Tu and M. C. Turner, "A novel robust disturbance rejection anti-windup framework," Int. J. Robust Nonlinear Control, vol. 84, no. 1, pp. 123-137, Jan. 2011.

[19] W. Sun, Z. Zhao, and H. Gao, "Saturated adaptive robust control fo active suspension systems," IEEE Trans. Ind. Electron., vol. 60, no. 9, pp. 3889-3896, Sep. 2013.

[20] O. Sörnmo, B. Olofsson, A. Robertsson, and R. Johansson, "Adaptive internal model control for mid-ranging of closed-loop systems with internal saturation intelligent," in Proc. IEEE/RSJ Int. Conf. Robots Syst., Tokyo, Japan, Nov. 2013, pp. 4893-4899.

[21] S. Gayadeen and S. R. Duncan, "Discrete-time anti-windup compensation for synchrotron electron beam controllers with rate constrained actuators,' Automatica, vol. 67, pp. 224-232, May 2016.

[22] X. Song, P. K. Gillella, and Z. Sun, "Low-Order stabilizer design for discrete linear time-varying internal model-based system," IEEE/ASME Trans. Mechatronics, vol. 20, no. 6, pp. 2666-2677, Dec. 2015.

[23] Z. Zhang, P. Yan, H. Jiang, and P. Ye, "A discrete time-varying internal model-based approach for high precision tracking of a multi-axis servo gantry," ISA Trans., vol. 53, no. 5, pp. 1695-1703, Sep. 2014

[24] T. Hu, A. R. Teel, and L. Zaccarian, "Anti-windup synthesis for linear control systems with input saturation: Achieving regional, nonlinear performance," Automatica, vol. 44, no. 2, pp. 512-519, Feb. 2008

[25] P. Liu, P. Yan, Z. Zhang, and T. Leng, "Modeling and control of a novel $\mathrm{X}-\mathrm{Y}$ parallel piezoelectric-actuator driven nanopositioner," ISA Trans. vol. 56, pp. 145-154, May 2015.

[26] J. Doyle, B. Francis, and A. Tannenbaum, Feedback Control Theory. New York, NY, USA: Macmillan, 1992.

[27] V. M. Popov, "Absolute stability of nonlinear systems of automatic control," Autom. Remote Control, vol. 22, no. 8, pp. 857-875, Mar. 1962.

[28] H. K. Khalil, Nonlinear Systems. Englewood Cliffs, NJ, USA: Prentice Hall, 1996

[29] P. Liu, P. Yan, Z. Zhang, and T. Leng, "Flexure-hinges guided nano-stage for precision manipulations: Design, modeling and control," Int. J. Precis. Eng. Manuf., vol. 16, no. 11, pp. 2245-2254, Oct. 2015.

[30] G. Y. Gu, L. M. Zhu, C. Y. Su, and H. Ding, "Motion control of piezoelectric positioning stages: Modeling, controller design and experimental evaluation," IEEE/ASME Trans. Mechatronics, vol. 18, no. 5, pp. 1459 1471 , Oct. 2013

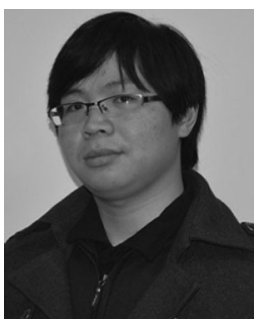

Pengbo Liu received the B.Eng. degree in mechanical engineering from the Ocean University of China, Qingdao, China, in 2012. He is currently working toward the Ph.D. degree in mechatronics engineering at Shandong University, Jinan, China.

His research interests include compliant mechanisms, micro/nanomanipulations, and their applications in industrial systems.

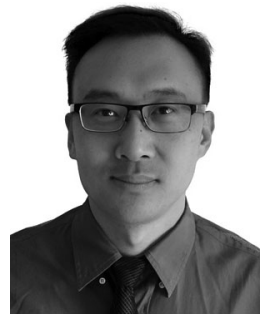

Peng Yan (M'03-SM'09) received the B.S. and M.S. degrees in electrical engineering from Southeast University, Nanjing, China, in 1997 and 1999, respectively, and the Ph.D. degree in electrical engineering from The Ohio State University, Columbus, OH, USA, in 2003

$\mathrm{He}$ is currently a full Professor with the School of Mechanical Engineering, Shandong University, Jinan, China, and also with the School of Automation Science and Electrical Engineering, Beihang University, Beijing, China. He has worked in various industry positions before joining Shandong University, including as a Staff Scientist at the United Technologies Research Center, East Hartford, CT, USA, from 2010 to 2011, and as a Senior Staff Engineer at Seagate Technology, Twin Cities, MN, USA, from 2005 to 2010. His current research interests include robust control, hybrid systems, and control of high-precision mechatronics. He has authored more than 70 scientific papers and more than 30 granted/pending patents.

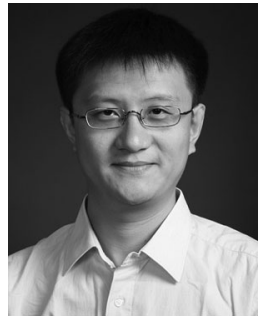

Zhen Zhang (S'06-M'08) received the B.S degree from Shanghai Jiao Tong University, Shanghai, China, in 1998, the M.S. degree from Tsinghua University, Beijing, China, in 2001, the M.S. degree from Vanderbilt University, Nashville, TN, USA, in 2003, and the Ph.D. degree in systems and control from The Ohio State University, Columbus, $\mathrm{OH}$, USA, in 2007.

From 2007 to 2009, he was a Postdoctoral Researcher with the Department of Mechanical Engineering, University of Minnesota, Minneapolis, MN, USA. Since 2009, he has been with the Department of Mechanical Engineering, Tsinghua University, Beijing, China, where he is currently an Associate Professor. His research interests include tracking of time-varying/parameter-varying systems, and design, modeling, and control of high-precision mechatronic systems and nanoscale positioning systems for advanced manufacturing applications. He has published more than 50 peer-reviewed technical articles in international journals and conference proceedings, and has 17 inventions either patented or patent pending.

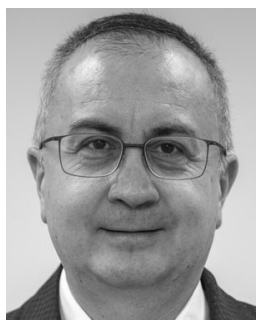

Hitay Özbay (M'89-SM'97) received the B.S. degree from Middle East Technical University, Ankara, Turkey, in 1985, the M.Eng. degree from McGill University, Montreal, QC, Canada, in 1987, and the Ph.D. degree from the University of Minnesota, Minneapolis, MN, USA, in 1989.

$\mathrm{He}$ is currently a Professor of electrical and electronics engineering, Bilkent University, Ankara, Turkey. $\mathrm{He}$ was with the University of Rhode Island (1989-1990) and The Ohio State University (OSU) (1991-2006), where he was a Professor of electrical and computer engineering, prior to joining Bilkent University in 2002, on leave from OSU. His research interests include robust control of distributed parameter systems. He has published three books, coedited one book, authored more than 200 refereed papers in edited books, journals, and conference proceedings.

Prof. Özbay has been active in the IEEE, International Federation of Automatic Control (IFAC), and Society for Industrial and Applied Mathematics (SIAM) conference organizations: Program Committee Member for CDCs 2001, 2005, 2009, 2011, and 2012, for IFAC World Congresses 2008, 2011, and 2014, for MTNS 2010, 2014, and Program Committee Cochair for SIAM CT2015. He served as an Associate Editor for the IEEE TRANSACtions on Automatic Control (1997-1999), Automatica (2001-2007), and SIAM Journal on Control and Optimization (20112015). He was an appointed member of the Board of Governors of the IEEE Control Systems Society (1999 and 2013), and a Vicechair of the IFAC Technical Committee on Networked Control Systems (2005-2011). $\mathrm{He}$ is currently on the Editorial Board of Automatica (since 2012) and the Springer book series Advances in Delays and Dynamics (since 2013). $\mathrm{He}$ is elected as a General Assembly Member of The European Control Association representing Turkey. 